\begin{abstract}
Alexandru Cristian ION
E-mail:cristianionmed@yahoo.com

Ştefan BUSNATU

E-mail: stefan.busnatu@umfed.ro

Carol Davila University of Medicine and Pharmacy Bucharest

BagdasarArseni Emergency Clinical Hospital Bucharest

Cardiology Department

Cristinel Dumitru BADIU

E-mail: cristinel.badiu@umfed.ro

Carol Davila University of Medicine and Pharmacy Bucharest

BagdasarArseni Emergency Clinical Hospital Bucharest

General Surgery Department

Roxana-VioletaPARTAS-CIOLAN, PhD Student

E-mail: roxana.partas@gmail.com

The Bucharest University of Economic Studies

Crina Julieta SINESCU

E-mail: crina.sinescu@gmail.com

Associate Professor, Cătălina Liliana ANDREI, PhD

E-mail: catalina.andrei@umfed.ro (Corresponding author)

Carol Davila University of Medicine and Pharmacy Bucharest

BagdasarArseni Emergency Clinical Hospital Bucharest

Cardiology Department
\end{abstract}

\title{
EVOLUTION OF RIGHT VENTRICULAR DYSFUNCTION MARKERS IN PATIENTS WITH INTERMEDIATE-HIGH RISK PULMONARY THROMBOEMBOLISM
}

\begin{abstract}
Intermediate-high risk pulmonary embolism is a life-threatening condition. Current evidence for the benefit of thrombolytic therapy in this condition is limited. In this study, we compared the effect of thrombolytic therapy and conventional therapy in patients with this condition. We did not find statistically significant differences between the two groups regarding gender-based distribution. The classically treated group presented a statistically significant older age, through the selection criteria, because age is the main factor associated with hemorrhagic reactions. We highlighted the statistically significant improvement of the right-
\end{abstract}

DOI: $10.24818 / 18423264 / 55.3 .21 .20$ 
Alexandru Cristian Ion, Ștefan Busnatu, Cristinel Dumitru Badiu

Roxana-Violeta Partas-Ciolan, Julieta Sinescu, Cătălina Liliana Andrei

ventricular dysfunction markers (ultrasound - Tricuspid Annular Plane Systolic Excursion (TAPSE), right-ventricle diameter (RV) - and biochemical - brain natriuretic peptide (BNP) -) in patients treated with thrombolytic therapy. The impact of the aforementioned markers on the clinical prognosis criteria - mortality - is obvious, considering the current evidence regarding their association with these markers.

Keywords: Pulmonary embolism, thrombolytic therapy, conventional therapy, right-ventricular dysfunction markers.

\section{JEL Classification: A12, C18, C3, I10}

\section{Introduction}

Venous thromboembolism (VTE), clinically presented in the form of deep vein thrombosis (DVT) or pulmonary embolism (PE), is globally the third most frequent cardiovascular emergency after myocardial infarction and stroke (1). Analyzes by age groups show that the incidence of VTE is 8 times higher in individuals in the age group over 80 years as compared to the age group 41-50 years (2). In parallel, longitudinal studies have shown an increasing incidence of VTE over time, in parallel with increasing frequency of risk factors (3). Death rates in EP are declining compared to previous decades $(2,3)$.Regarding the risk factors for VTE, major traumas, major surgeries, lower limb fractures and hip prostheses, severe spinal injuries, recent myocardial infarction - 3 months ago -, recent paroxysmal atrial fibrillation or flutter episodes - in the last 3 months - are strong predisposing factors (with increased risk of VTE more than 10 times) (4). Neoplasia is a recognized risk factor for VTE, the highest risk being associated with pancreatic, gastric, cerebral and haematological neoplasms $(5,6)$.

\section{Data and methodology}

The aim of the current study is to analyze the dynamics of right ventricular overload markers in a comparative manner in patients with intermediate-high risk PE who are thrombolyzed and treated classically. The importance of the objective results from the proven correlation between the markers of right ventricular dysfunction and the prognostic clinical indices - mortality and hemodynamic instability.

In this study we included patients with acute PE diagnosed by contrastenhanced thoracic CT, with intermediate-increased risk, characterized by a clinical severity score PESI $>60$, ultrasound RV dysfunction (revealed either by RV dilation due to pressure overload RV, by longitudinal RV systolic dysfunction (assessed by TAPSE) $(7,8)$ or by decrease in contractile myocardial velocity (MCV)) and RV 
Evolution of Right Ventricular Dysfunction Markers in Patients with IntermediateHigh Risk Pulmonary Thromboembolism

dysfunction revealed by biological samples (BNP $>150 \mathrm{pg} / \mathrm{ml})$. Included patients must have inclusion criteria and no exclusion criteria. The inclusion criteria are: 1 . the first episode of acute pulmonary embolism in life; 2 . persons over 18 years of age; 3 . persons who understand the study and agree to be part of it - informed consent. Exclusion criteria: 1. Increased risk of bleeding secondary to blood disorders: hemophilia, severe thrombocytopenia $<50,000 / \mathrm{mm} 3 ; 2$. Severe systolic dysfunction of the left ventricle assessed by LV ejection fraction $<30 \%$; 3 . Severe valvulopathy possibly associated with secondary pulmonary hypertension: tight mitral stenosis, significant mitral regurgitation, tight aortic stenosis, severe aortic insufficiency; 4. Previous pulmonary embolism; 5 . the presence of diseases that limit life expectancy to less than 1 year: terminal neoplasms; 7. Persons incapable of exercising informed consent; 8 . Age over 85 years. We included in this study 82 consecutive patients with PE with intermediate-high risk defined according to the ESC criteria, respecting the inclusion and exclusion criteria $(9,10)$. Patients over the age of 85 were excluded from the study due to increased bleeding risks, which were shown to be associated with anticoagulant and thrombolytic therapy. Patients were divided into 2 groups, study group 39 patients (receiving thrombolytic therapy t-PA 100mg in 2 hours combined with anticoagulant therapy - unfractionated heparin (HNF) dosed according to the partialthromboplastin time (aPTT) and the control group - 43 patients (treated with HNF dosed according to aPTT). The control group included patients with contraindications for thrombolytic therapy and patients in whom, according to current evidence, thrombolytic therapy is associated with increased risk of bleeding (obese $(\mathrm{BMI}>30 \mathrm{~kg} / \mathrm{m} 2)$ and underweight $(\mathrm{BMI}<18.5 \mathrm{~kg} / \mathrm{m} 2)$, severe renal dysfunction $\mathrm{ClCre}<30 \mathrm{ml} / \mathrm{min} / \mathrm{m} 2-$-). Patients were evaluated upon admission, in terms of the clinical severity of PE, by the PESI score, as well as in terms of the incidence of the main risk factors (neoplasia, hip fracture, recent hip prosthesis, major trauma, major surgery), the presence of chronic medical conditions - chronic obstructive pulmonary disease (COPD), heart failure (CHF)). Evaluation of RV overload markers included BNP dosing at admission, evaluation of echocardiographic signs of RV overload (RV dilation, supraunitary RV/LV ratio, TAPSE, VCM) $(11,12,13,14)$. Subsequently, at $48 \mathrm{~h}$ the echocardiographic re-evaluation followed the dynamics of the same markers, and the BNP redose at $72 \mathrm{~h}$ followed the evolution of BNP. Statistical analysis was performed using SPSS, and the use of Chi-square statistical significance tests, Welch T test, Wilcoxon test and Mann-Whitney $(15,16,17)$ test depending on the normality of data distribution - evaluated by the Shapiro-Wilk test.

\section{Results}

The statistical analysis consisted of 4 stages. A first part regarding the analysis of the distribution by age groupsand by genders in the two groups, the study group and 
Alexandru Cristian Ion, Ștefan Busnatu, Cristinel Dumitru Badiu

Roxana-Violeta Partas-Ciolan, Julieta Sinescu, Cătălina Liliana Andrei

the control group. We also analyzed the distribution of the main risk factors in the two groups, comparatively.

Regarding the analysis of the distribution by age groups, the elements of descriptive statistics show an average age of 61.85 years in the study group compared to 71.28 years in the control group. This difference can be justified by the fact that the incidence of contraindications for thrombolytic therapy increases with age, which has led to the inclusion of older patients in the non-thrombolytic group.

Table 1: Distribution of the average age in the two study and control groups

Descriptive Statistics

\begin{tabular}{|c|c|c|}
\hline & \multicolumn{2}{|c|}{ Age } \\
\hline & Study & Control \\
\hline Valid & 39 & 43 \\
\hline Missing & 0 & 0 \\
\hline Mean & 61.85 & 71.28 \\
\hline Median & 64.00 & 74.00 \\
\hline Fashion & 68.00 & 77.00 \\
\hline Std. Deviation & 13.09 & 10.29 \\
\hline IQR & 16.50 & 13.50 \\
\hline Minimum & 24.00 & 43.00 \\
\hline Maximum & 81.00 & 89.00 \\
\hline
\end{tabular}

Regarding the distribution by age groups, there is a higher weight of patients in the 6070 age group in the study group compared to a higher share of the 70-80 age group in the control group.

To analyze the statistical significance of the difference between the mean ages of the two groups, we first analyzed whether the data are normally distributed, using the Shapiro-Wilk normality test and the Levene equal variation test.

Table 2: Analysis of equal data distribution by Levene test

Test of Normality (Shapiro-Wilk)

\begin{tabular}{llll}
\hline & & W & $\mathrm{p}$ \\
\hline Age & Study & 0.93 & 0.02 \\
& Control & 0.96 & 0.11 \\
\hline
\end{tabular}

Given the unequal distribution according to the Levene test, we performed the Welch $t$ test to determine if there was a statistically significant difference between the two groups in terms of age. Considering the value $p<0.001$, the result is statistically 
Evolution of Right Ventricular Dysfunction Markers in Patients with IntermediateHigh Risk Pulmonary Thromboembolism

significant, the patients from the study group presenting a statistically significantly lower average age than those from the control group.

Table 3: Welch test on the statistical significance of the age difference between the two groups

Independent Samples T-Test

\begin{tabular}{llll}
\hline & $\mathrm{t}$ & $\mathrm{df}$ & $\mathrm{p}$ \\
\hline Age & -3.60 & 72.05 & $<.001$ \\
\hline
\end{tabular}

In terms of gender distribution in the study group, the frequency of females was $53.85 \%$ compared to $58.14 \%$ in the control group. Subsequently, we used the Chisquare test, without showing a statistically significant difference in terms of distribution by sex ( $p$ value 0.7 ).

A second element of the statistical analysis was the analysis of the average PESI score, with an average PESI score of 94.8 in the study group compared to 108 in the control group, statistically significant difference ( $\mathrm{P}$ 0.02). This difference is the result of selection criteria, considering that the average age of the control group was statistically significantly higher than that of the study group, the share in the PESI score of the patient's age being important. At the same time, some determinants of the PESI score had a higher weight in the control group compared to the study group, these factors being correlated with the age of the patients. Thus, TS disorientation was present in $12.82 \%$ in the study group, compared to $32.55 \%$ in the control group.

Table 4: Contingency table regarding the distribution of temporospatial disorientation at admission in the two groups. Analysis of the statistical significance of the difference regarding the temporospatial disorientation by the Chi-square test

\begin{tabular}{|l|l|l|l|}
\hline Group & Yes & Not & Total \\
\hline Study & 5 & 34 & 39 \\
\hline Control & 14 & 29 & 43 \\
\hline Total & 19 & 63 & 82 \\
\hline
\end{tabular}

For the rest of the determinants of the PESI score, no statistically significant differences were identified between the two groups. However, considering the fact that age is an additive element and temporospatial disorientation is the most important determinant (60 points in the PESI score) it is easy to explain the statistically significant difference between the two groups regarding the average PESI score. 
Alexandru Cristian Ion, Ștefan Busnatu, Cristinel Dumitru Badiu

Roxana-Violeta Partas-Ciolan, Julieta Sinescu, Cătălina Liliana Andrei

Regarding the risk factors for VTE, we analyzed the distribution of the main risk factors (neoplasms, recent hip fractures, recent hip prostheses) in the two groups. Neoplasia was present in $35.9 \%$ in the study group compared to $41.86 \%$ in the control group.

Table 5: Table on the frequency of distribution of neoplasms in the two groups Frequencies for FR neoplasia

\begin{tabular}{|c|c|c|c|c|c|}
\hline \multirow{2}{*}{$\frac{\text { Group }}{\text { Study }}$} & \multicolumn{5}{|c|}{ FR neoplasia Frequency Percent Valid Percent Cumulative Percent } \\
\hline & Yes & 14 & 35.90 & 35.90 & 35.90 \\
\hline & Not & 25 & 64.10 & 64.10 & 100.00 \\
\hline & Missing & 0 & 0.00 & & \\
\hline & Total & 39 & 100.00 & & \\
\hline Control & Yes & 18 & 41.86 & 41.86 & 41.86 \\
\hline & Not & 25 & 58.14 & 58.14 & 100.00 \\
\hline & Missing & 0 & 0.00 & & \\
\hline & Total & 43 & 100.00 & & \\
\hline
\end{tabular}

Subsequently, by using the Chi-square test we analyzed the existence of statistically significant differences between the two groups regarding the frequency of neoplasia. We did not highlight statistically significant differences between the two groups ( $p$ value 0.58 )

Table 6: Chi-square test for the frequency of neoplasia in the two groups, without statistically significant differences

\begin{tabular}{|l|l|l|l|}
\hline & Value & df & $\mathrm{p}$ \\
\hline $\mathrm{X}^{2}$ & 0.31 & 1 & 0.58 \\
\hline $\mathrm{N}$ & 82 & & \\
\hline
\end{tabular}

Similarly, the percentage of patients with recent hip prostheses in the study group was $23.08 \%$ compared to $25.58 \%$ in the control group, with no statistical significance of this difference ( $\mathrm{p}$ value 0.79 ). Regarding recent hip fractures, the percentage of patients with recent hip fractures was $23.08 \%$ in the study group, compared to $27.9 \%$ in the control group ( $\mathrm{p}$ value 0.62 ).

A final element of the statistical analysis was the comparative analysis of the variation of RV overload markers between the two groups. This element is essential, given the known correlation of markers of right ventricular dysfunction with mortality and hemodynamic instability. In the study group we could highlight a decrease of the average telediastolicRV diameter by $24.13 \%$ at the evaluation at 48 hours compared to 
Evolution of Right Ventricular Dysfunction Markers in Patients with IntermediateHigh Risk Pulmonary Thromboembolism

the admission. At the same time, in the control group the RVtelediastolic diameter decreased by $11.63 \%$ at 48 hours compared to the intake.

Table 7: Average values of RV upon admission and after $48 \mathrm{~h}$ in the study group and in the control group

\begin{tabular}{|l|l|l|l|l|l|l|l|l|l|}
\hline & $\mathrm{N}$ & Mean & $\mathrm{Sd}$ & $\mathrm{Se}$ & & $\mathrm{N}$ & Mean & $\mathrm{Sd}$ & $\mathrm{Se}$ \\
\hline $\begin{array}{l}\text { RV } \\
\text { admission }\end{array}$ & 39 & 47.79 & 4.86 & 0.78 & $\begin{array}{l}\text { RV } \\
\text { admission }\end{array}$ & 42 & 44.33 & 4.98 & 0.77 \\
\hline rv 48h & 39 & 36.23 & 4.01 & 0.64 & rv 48h & 42 & 39.17 & 5.60 & 0.86 \\
\hline \multicolumn{2}{|l|}{ Study group } \\
\hline
\end{tabular}

Subsequently, the distribution of RV diameter values is presented graphically in Table 2, and an increased frequency of RV dilation (telediastolic diameter over 40 $\mathrm{mm}$ ) can also be observed in the study group and in the control group. This element is completely justified by the acute pressure RV overload, acutely installed, which has as an adaptive mechanism the acute dilation of the right ventricle.

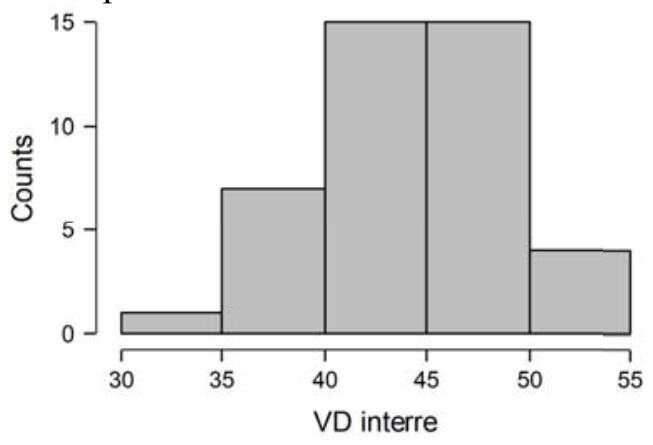

Study group

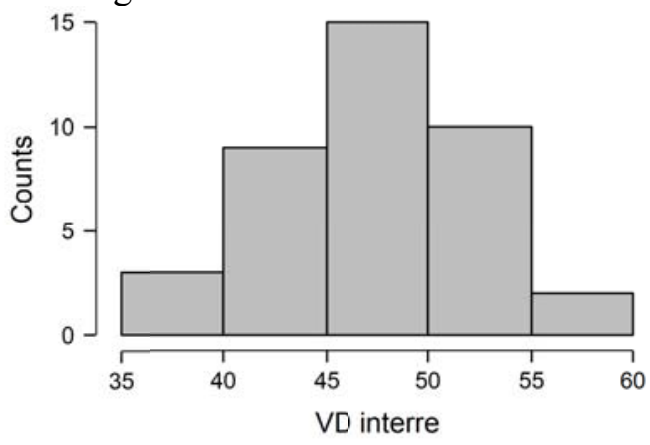

Control group

\section{Graph1: Distribution of RV diameters at admission in the study group and in the control group}

Both in the study group and in the control group a statistically significant reduction of $\mathrm{RV}$ diameters was identified at $48 \mathrm{~h}$ compared to admission, this variation being statistically significant in both groups $(24.13 \%$, p value $<0.001$ in the study group vs $11.63 \%$, p value $<0.001$ in the control group). This variation is completely justified considering that following the applied therapy the overload pressure on the RV is reduced, and in this way the adaptive mechanism represented by the acute dilation of 
Alexandru Cristian Ion, Ștefan Busnatu, Cristinel Dumitru Badiu

Roxana-Violeta Partas-Ciolan, Julieta Sinescu, Cătălina Liliana Andrei

the RV is canceled. Associated with the reduction of the RV diameter the parietal stress is also reduced, which varies directly proportional to the diameter of the cavity, as well as the myocardial oxygen consumption, the result being the improvement of the RV contractility parameters.

Subsequently, we analyzed whether there are statistically significant differences between the variations of RV diameters between the two groups. Given that they were also not distributed normally and homogeneously (according to the Shapiro-Wilk test) for the comparative analysis we used a non-parametric test (Mann-Whitney test).

Table 8: Shapiro-Wilk and Mann-Whitney tests on differences between RV diameter variations between study group and control group

\begin{tabular}{|l|l|l|l|}
\hline & & W & p \\
\hline difference & Study & 0.95 & 0.08 \\
\cline { 2 - 4 } & Control & 0.96 & 0.14 \\
\hline
\end{tabular}

Note. Significant results suggest a deviation from normality

In conclusion, in the study group the variation of RV diameters is statistically significantly more important than in the control group, an element that supports the beneficial effects of thrombolytic therapy on RV pressure overload in acute pulmonary embolism compared to classical therapy.

Subsequently, we analyzed the TAPSE variation in each separate group, at 72 hours compared to the level upon admission. Regarding this parameter, it is correlated with $\mathrm{RV}$ kinetics. Under conditions of RV overload, myocardial oxygen consumption of the right ventricular myocardium will increase due to increased parietal stress. The result is an imbalance between myocardial (fixed) oxygen intake and increased myocardial oxygen consumption, which causes myocardial ischemia with altered RV kinetics. The affected echocardiographic marker is represented by the decrease of TAPSE, and subsequently, following the decrease of the pressure in the pulmonary artery and of the RV post-pregnancy, the myocardial oxygen consumption of RV will decrease and the RV kinetics will be recovered, with the return of TAPSE and of the myocardial contraction velocity at the level of the RV myocardium.

In the study group there is an increase in TAPSE at 48 hours by $48.92 \%$. To identify the statistical significance of this difference we evaluated the normal distribution of data by the Shapiro-Wilk test. Given that the test did not show a normal distribution of data, we evaluated the statistical significance of the TAPSE variation in the study 
Evolution of Right Ventricular Dysfunction Markers in Patients with IntermediateHigh Risk Pulmonary Thromboembolism

group by the Wilcoxon test of pair ranks, which highlights the statistical significance of the TAPSE increase 48 hours after admission to the study group ( $p$ value $<0.001$ ).

Table 9: Shapiro-Wilk and Wilcoxon tests on TAPSE variation in the study group, between admission and $48 \mathrm{~h}$

\begin{tabular}{|l|l|l|l|}
\hline & & W & $\mathrm{p}$ \\
\hline TAPSE admission & TAPSE 48h & 0.92 & 0.01 \\
\hline Note. Significant results suggest a deviation from normality \\
\hline \multicolumn{2}{|l|}{ Paired Samples t-Test } & W & $\mathrm{p}$ \\
\hline & & 14 & 0.00 \\
\hline TAPSE admission & TAPSE 48h &
\end{tabular}

Note. Wilcoxon signed-rank test.

In the control group, the statistical analysis followed the same steps, in order to evaluate the TAPSE variation 48 hours after admission, as well as its statistical significance. We identified an increase in TAPSE at 48 hours in the control group of $23.47 \%$ compared to admission. For the analysis of the statistical significance we used the Shapiro-Wilk test to evaluate the normality of the data distribution. Given that the data did not have a normal distribution for the statistical significance of TAPSE variation in the control group we used a nonparametric test, the Wilcoxon test of the paired ranks, highlighting a significant increase in TAPSE at 48 hours in the study group compared to admission ( $p$ value $<0.001$ ).

Table 10: Shapiro-Wilk and Wilcoxon tests on TAPSE variation in the control group, between admission and $48 \mathrm{~h}$

\begin{tabular}{|l|l|l|l|}
\hline \multicolumn{4}{|l|}{ Test of Normality (Shapiro-Wilk) } \\
\hline & & W & p \\
\hline TAPSE admission & TAPSE 48h & 0,74 & 0.001 \\
\hline
\end{tabular}

Subsequently, we analyzed whether the difference between the TAPSE variation upon admission and $48 \mathrm{~h}$ in the study group and the control group is statistically significant. Table 11 presents descriptively the TAPSE variations in the 2 groups, with the associated standard derivations. 
Alexandru Cristian Ion, Ștefan Busnatu, Cristinel Dumitru Badiu

Roxana-Violeta Partas-Ciolan, Julieta Sinescu, Cătălina Liliana Andrei

Table 11: Descriptive table regarding the TAPSE variations between the 2 groups study group and control group

\begin{tabular}{|l|l|l|l|l|l|}
\hline & Group & N & Mean & SD & SE \\
\hline \multirow{2}{*}{ Dif TAPSE } & Study & 39 & 5.72 & 3.32 & 0.53 \\
\cline { 2 - 6 } & Control & 41 & 3.39 & 4.63 & 0.72 \\
\hline
\end{tabular}

Considering the abnormal data distribution, according to the Shapiro-Wilk test and the Levine test, we opted to analyze the statistical significance of the difference between TAPSE variations in the two groups (at 48 hours compared to admission) for the Welch $t$ test.

Table 12: Shapiro-Wilch test for normal distribution of data on TAPSE variation Test of Normality (Shapiro-Wilk)

\begin{tabular}{|l|l|l|l|}
\hline & & W & p \\
\hline Dif TAPSE & Study & 0.92 & 0.01 \\
\hline & Contro & 0.74 & 0.001 \\
\hline
\end{tabular}

Note. Significant results suggest a deviation from normality

We analyzed by $t$ Welch test (nonparametric test) the statistical significance of the difference between TAPSE variations in the 2 groups (TAPSE increase at 48 hours compared to admission with $48.92 \%$ in the study group vs. $23.47 \%$ in the control group). The Welch test demonstrated the statistical significance of this difference between variations ( $\mathrm{p}$ value 0.01 ), an element that further supports the beneficial effect of thrombolytic therapy on RV contractility. This element is justified by the additional decrease of post-load by thrombolytic therapy compared to classical therapy, in this way the myocardial oxygen consumption in the RV myocardium being reduced and the RV kinetics being recovered.

Table 13: $t$ Welch test on the statistical significance of the differences between the TAPSE variations between the 2 groups

\begin{tabular}{|l|l|l|l|}
\hline \multicolumn{4}{|l|}{ Independent Samples T-Test } \\
\hline & $\mathrm{t}$ & $\mathrm{df}$ & $\mathrm{p}$ \\
\hline Dif TAPSE & 2.59 & 72.64 & 0.01 \\
\hline
\end{tabular}

Note. Welch's test

A last element analyzed among the imaging RV overload markers was the myocardial contraction velocity (MCV) at the RV free wall level. Echocardiography is evaluated by tissue Doppler (TDI) method, the myocardial contraction velolcity(S) being the first altered marker in case of a contractile RV dysfunction. We identified an increase in $\mathrm{S}$ 
Evolution of Right Ventricular Dysfunction Markers in Patients with IntermediateHigh Risk Pulmonary Thromboembolism

wave velocity by $58.88 \%$ at 48 hours compared to admission in the study group, in order to test the statistical significance of this difference using the Wilcoxon test of pair ranks, considering the abnormal distribution of data (according to the ShapiroWilch test).

Table 14: Shapiro-Wilk and Wilcoxon tests for S-speed variations in the 48-hour study group compared with admission

\begin{tabular}{|c|c|c|c|}
\hline \multicolumn{2}{|c|}{ Test of Normality (Shapiro-Wilk) } & $\mathrm{W}$ & $p$ \\
\hline $\mathrm{S}$ admission & $\mathrm{S} 48 \mathrm{~h}$ & 0.92 & 0.01 \\
\hline \multicolumn{4}{|c|}{ Note. Significant results suggest a deviation from normality } \\
\hline \multicolumn{4}{|c|}{ Paired Samples T-Test } \\
\hline & & $\mathrm{W}$ & $\mathrm{p}$ \\
\hline $\mathrm{S}$ admission & $\mathrm{S} \mathrm{48h}$ & 0.00 & 0.01 \\
\hline
\end{tabular}

Note. Wilcoxon signed-rank test.

The Wilcoxon test demonstrated the statistical significance of the increase in myocardial contraction velocity at 48 hours as compared to admission to the study group ( $\mathrm{p}$ value $<0.001$ ).

In the control group we identified an increase in myocardial contraction velocity by $27.92 \%$ at 48 hours compared to admission. Subsequently, we used the Shapiro-Wilk test to test the normality of the data distribution. Through the abnormal distribution of the data we used the nonparametric Wilcoxon test of the pair ranks. The variation of speed S was statistically significant at 48 hours compared to admission in the control group ( $\mathrm{p}$ value $<0.001$ ).

There can be a difference between the variation of the S speed at 48 hours in the study group compared to the control group (58.88\% study group vs $27.92 \%$ control group). We analyzed the statistical significance of this difference by the $t$ Welch test considering the unequal distribution of data according to the Shapiro-Wilch test Consistent with the variation of TAPSE and RV telediastolic diameters the variation of $\mathrm{S}$ wave velocity was more important in the thrombolyzed group compared to the control group $(\mathrm{p}<0.001)$. The fact that the improvement of all RV pressure overload imaging parameters was more important in the study group compared to the control group supports the beneficial effect of thrombolytic therapy compared to classical therapy. In addition, the improvement of imaging markers is associated with prognostic clinical indices (mortality and hemodynamic instability), an element that further supports the positive effect of thrombolytic therapy compared to conventional therapy in patients with intermediate-high risk PE. 
Alexandru Cristian Ion, Ștefan Busnatu, Cristinel Dumitru Badiu

Roxana-Violeta Partas-Ciolan, Julieta Sinescu, Cătălina Liliana Andrei

Regarding the biological markers of RV overload, we analyzed the evolution of BNP at 72 hours compared to the admission in the two groups. In the study group we identified a decrease in BNP values at $72 \mathrm{~h}$ compared to admission by $80.67 \%$, and the analysis of statistical significance was performed by the Wilcoxon test of the pair ranks (given the abnormal distribution of data). The Wilcoxon test showed that the decrease in BNP at 72 hours was statistically significant compared to BNP at admission in the study group ( $\mathrm{p}$ value $<0.001$ ).

Table 15: Descriptive presentation of BNP values at admission and at 72 hours as well as the Wilcoxon test on the statistical significance of BNP decrease at 72 hours in the study group

Descriptive

\begin{tabular}{lllcl}
\hline & $\mathrm{N}$ & Mean & SD & SE \\
\hline BNPadmission & 39 & 7334.87 & 6213.52 & 994.96 \\
BNP 72h & 37 & 1417.24 & 1884.08 & 309.74 \\
\hline
\end{tabular}

In the control group, the variation of BNP at $72 \mathrm{~h}$ compared to admission was evaluated, with a decrease of BNP values by $46.75 \%$ at $72 \mathrm{~h}$ compared to admission. The statistical significance of this variation was tested by the Wilcoxon test ( $p$ value $<0.001)$.

Table 16: Descriptive presentation of BNP values at admission and at 72 hours in the control group

\begin{tabular}{|l|l|l|l|l|}
\hline & $\mathrm{N}$ & Mean & SD & SE \\
\hline BNP admission & 43 & 5439.19 & 6566.81 & 1001.43 \\
\hline BNP 72h & 38 & 2896.42 & 4188.51 & 679.47 \\
\hline
\end{tabular}

Therefore, regarding the BNP variation at $72 \mathrm{~h}$ compared to admission, in the study group we can see a decrease by $80.67 \%$ compared to $46.75 \%$ in the control group. We analyzed the statistical significance of this difference between the BNP variations by the Welch $t$ test, highlighting a statistically significant difference ( $p$ value $<0.001$ ). This result is consistent with the evolution of RV dysfunction imaging markers, supporting the additional hemodynamic improvement induced by thrombolytic therapy compared to unfractionated heparin therapy. 
Evolution of Right Ventricular Dysfunction Markers in Patients with IntermediateHigh Risk Pulmonary Thromboembolism

\section{Conclusions and discussions}

Thrombolytic therapy is associated, unlike conventional therapy, to a higher rate of repermeabilization of the pulmonary vessels. The result is a decrease in pulmonary vascular resistance, with a decrease in RV postload. The directly proportional relation, according to Laplace's law, between the parietal stress and the intracavitary pressure and the diameter of the cavity, explains why in the conditions of the RV posloaddecrease, the parietal stress will decrease, due to the decrease of the intracavitary pressure. At the same time, the diameter of the RV will decrease, given that the acute expansion is initially mobilized as an adaptive mechanism to the overload of the RV pressure to increase the contractile force (according to the FrankStarling law).

The elements that determine the decrease of parietal stress will similarly determine a decrease of myocardial oxygen consumption. The balance between oxygen demand in the RV myocardium (renormalized) and constant myocardial oxygen supply explains the improvement in RV kinetics, which appeared after the decrease in postload following reperfusion therapy. The pathophysiological bases of the improvement of RV dysfunction parameters are justified, but the evidence regarding the benefit compared to classical therapy is limited, the extensive evaluation of all hemodynamic and imaging parameters to support the mentioned pathophysiological model being necessary.

In the current study, the patients were divided into 2 groups, study group thrombolyzed - and control group -conventional treatment -. According to the inclusion criteria, only patients with the first episode of acute PE with intermediatehigh risk (started in the last 2 weeks) were selected in the study. At the same time, due to multiple bleeding side effects, we excluded elderly patients - over 85 years of age as well as patients with severe anemic syndromes from the study. In the control group we included obese, underweight patients, as well as patients with severe renal dysfunction (Creatinine Clearance $<30 \mathrm{ml} / \mathrm{min} / \mathrm{m} 2$ ), an element supported by current evidence that thrombolytic therapy is associated with increased hemorrhagic risk for these subcategories. Also, patients with contraindications for thrombolytic therapy were included in the control group. In this way, the statistically significant difference between the average ages in the two groups is justified (61.85 years study group vs 71.28 years control group, $p$ value $<0.001$ ). Considering the weight of age in the PESI additive score for stratification of the clinical risk of PE, the statistically significant difference between the average PESI score of the two groups is also supported (94.8 study group vs 108 control group, $p$ value 0.02 ). By analyzing the gender distribution of the two groups, we did not identify statistically significant differences. In conclusion, according to the selection criteria, the patients subjected to classical 
Alexandru Cristian Ion, Ștefan Busnatu, Cristinel Dumitru Badiu

Roxana-Violeta Partas-Ciolan, Julieta Sinescu, Cătălina Liliana Andrei

therapy (HNF) were older patients and with a higher clinical severity score, for this category thrombolytic therapy being associated with major hemorrhagic risks.

By evaluating the impact of the two types of therapies on the markers of acute RV pressure overload and those of RV dysfunction (both imaging and biological) we wanted to evaluate the benefit of the two proposed schemes of therapy on the functional parameters of RV. For each group, both imaging parameters (telediastolic diameter RV, TAPSE, MCV) and biochemical parameters (BNP) showed a statistically significant improvement at reassessment at 48 hours (for echocardiographic markers) or $72 \mathrm{~h}$ for biochemical markers. However, the variation of the parameters in the study group was statistically significantly more important as compared to the control group. The BNP variation in the study group was $80.67 \%$ as compared to a decrease by $46.75 \%$ in the control group (statistically significant difference $p$ value $<0.001$ ). Also, the TAPSE variation was statistically significantly more important in the study group as compared to the control group (increase by $48.92 \%$ in the study group vs. $23.47 \%$ in the control group, $\mathrm{p}$ value 0.01 ). By analyzing the MCVvariation, we highlighted a statistically significant increase in the study group compared to the control group $(58.88 \%$ vs $27.92 \%$, p value $<0.001)$. The evolution of the RV telediastolic diameter at 48 hours after admission in the study group was statistically significantly more important than in the control group (decrease by $24.13 \%$ in the study group compared to $11.63 \%$ in the control group, $\mathrm{p}$ value $<0.001)$.

Considering that all biochemical and imaging markers are associated with prognosis clinical parameters (acute mortality and hemodynamic instability), it is obvious that the benefit of thrombolytic therapy on these markers can be extended to clinical parameters (this evaluation is the basis for a later study). The main limitation for the use of thrombolytic therapy was the associated increased hemorrhagic risk, but through the correct selection of patients and the identification of subgroups with high hemorrhagic risk, this adverse reaction was combated.

It is necessary to further evaluate the effects of thrombolytic therapy on the parameters of right ventricular dysfunction by including patients in the study, which may support the strength of evidence on improving the prognosis of subjects treated with thrombolytic therapy as compared to conventional therapy. A limitation of the current study may be the low number of patients, but this is due to inclusion criteria, elderly patients with high risk of bleeding being excluded.

Another discussion refers to the methods of evaluation of RV dysfunction, considering the fact that transthoracic echocardiography presents limits in the evaluation of RV functionality due to the anatomical peculiarities of RV. New echocardiographic evaluation methods (3D echocardiography) that evaluate functional RV parameters 
Evolution of Right Ventricular Dysfunction Markers in Patients with IntermediateHigh Risk Pulmonary Thromboembolism

volumetrically require a long time for purchase and are not standardized for evaluation. At the same time, the use of new imaging techniques, angio-CT and angio MRI for RV functionality would require a long time for image processing and, in this way, a limitation of their use in emergency mode would occur.

Currently, evidence on the effects of thrombolytic therapy in patients with high intermediate risk of PE is limited, with no efficacy on mortality, with an increased risk of bleeding. Given the existing evidence, it is necessary to stratify patients with PE with high intermediate risk who can benefit from thrombolytic therapy based on the risk of bleeding. Additional data are needed for a more accurate analysis of the impact of thrombolytic therapy in this category of patients.

\section{REFERENCES}

[1] Raskob G.E., Angchaisuksiri P., Blanco A.N., Buller H., Gallus A., Hunt B.J., Hylek E.M., Kakkar A., Konstantinides S.V., McCumber M., Ozaki Y., Wendelboe A., Weitz J.I. (2014),Thrombosis: A Major Contributor to Global Disease Burden. ArteriosclerThrombVascBiol2014;34:2363-2371;

[2] Wendelboe A.M., Raskob G.E.(2016),Global Burden of Thrombosis: Epidemiologic Aspects. Circ Res 2016;118:1340-1347;

[3] de Miguel-Diez J., Jimenez-Garcia R., Jimenez D., Monreal M., Guijarro R., Otero R., Hernandez-Barrera V., Trujillo-Santos J., Lopez de Andres A., Carrasco-Garrido P. (2014), Trends in Hospital Admissions for Pulmonary Embolism in Spain from 2002 to 2011. EurRespir J 2014;44:942-950;

[4] Rogers M.A., Levine D.A., Blumberg N., Flanders S.A., Chopra V., Langa K.M.(2012),Triggers of Hospitalization for Venous Thromboembolism. Circulation 2012;125:2092-2099;

[5] Timp J.F., Braekkan S.K., Versteeg H.H., Cannegieter S.C. (2013),Epidemiology of Cancer-associated Venous Thrombosis. Blood 2013;122:1712-1723;

[6] Blom J.W., Doggen C.J., Osanto S., Rosendaal F.R.(2005),Malignancies, Prothrombotic Mutations, and the Risk of Venous Thrombosis. JAMA 2005;293:715-722;

[7] Wattanakit K., Lutsey P.L., Bell E.J., Gornik H., Cushman M., Heckbert S.R., Rosamond W.D., Folsom A.R.(2012), Association between Cardiovascular Disease Risk Factors and Occurrence of Venous Thromboembolism. A Time-Dependent Analysis. ThrombHaemost2012;108:508-515; 
Alexandru Cristian Ion, Ștefan Busnatu, Cristinel Dumitru Badiu Roxana-Violeta Partas-Ciolan, Julieta Sinescu, Cătălina Liliana Andrei

[8] Stavros V.Konstantinides, Guy Meyer et al.(2020),2019 ESC Guidelines for the Diagnosis and Management of Acute Pulmonary Embolism Developed in Collaboration with the European Respiratory Society (ERS): The Task Force for the Diagnosis and Management of Acute Pulmonary Embolism of the European Society of Cardiology (ESC).European Heart Journal, Volume 41, Issue 4, 21 January 2020, Pages 543-603;

[9] Roy P.M., Colombet I., Durieux P., Chatellier G., Sors H., Meyer G.(2005), Systematic Review and Meta-Analysis of Strategies for the Diagnosis of Suspected Pulmonary Embolism. BMJ 2005;331:259;

[10] Bova C., Greco F., Misuraca G., Serafini O., Crocco F., Greco .A, Noto A.(2003),Diagnostic Utility of Echocardiography in Patients with Suspected Pulmonary Embolism.Am J Emerg Med 2003;21:180-183;

[11] Kurnicka K., Lichodziejewska B., Goliszek S., Dzikowska-Diduch O., Zdonczyk O., Kozlowska M., Kostrubiec M., Ciurzynski M., Palczewski P., Grudzka K., Krupa M., Koc M., Pruszczyk P.(2016),Echocardiographic Pattern of Acute Pulmonary Embolism: Analysis of 511 Consecutive Patients. J Am SocEchocardiogr2016;29:907-913;

[12]Becattini C., Agnelli G., Vedovati M.C., Pruszczyk P., Casazza F., Grifoni S., Salvi A., Bianchi M., Douma R., Konstantinides S., Lankeit M., Duranti M.(2011),MultidetectorComputed Tomography for Acute Pulmonary Embolism: Diagnosis and Risk Stratification in a Single Test. Eur Heart J2011;32:1657-1663; [13] Meinel F.G., Nance J.W.Jr., Schoepf U.J., Hoffmann V.S., Thierfelder K.M., Costello P., Goldhaber S.Z., Bamberg F.(2015),Predictive Value of Computed Tomography in Acute Pulmonary Embolism: Systematic Review and Meta-Analysis. Am J Med 2015;128:747-759;

[14]Coté B., Jimenez D., Planquette B., Roche A., Marey J., Pastre J., Meyer G., Sanchez O.(2017), Prognostic Value of Right Ventricular Dilatation in Patients with Low-Risk Pulmonary Embolism.EurRespir J 2017;50:1701611;

[15] Andrei C., Ciolan D.F., Mirica A., Toma I.A., Draganescu D., Radulescu F.S.(2019),Internship and the Next Generation of Farmacists in Romania.

Farmacia2019; 67 (2): 378-382;

[16] Ciolan D.F., Andrei C. , Mirica A., Toma I.A., Draganescu D., Radulescu F.S.(2020),Internships in Pharmaceutical Students' Development. Farmacia2020; 68(1):176-184;

[17] Drăgănescu D., Lupuleasa D., Dumitrescu.I B., DinuPârvu C. E., Ciolan D. F.(2013),Evidence on E-prescribing Systems Worldwide. First Romanian Results. Farmacia2013; 61(2):353-360. 